University of Wollongong

Research Online

Faculty of Engineering - Papers (Archive)

Faculty of Engineering and Information

Sciences

2011

\title{
The use of a silicon strip detector dose magnifying glass in stereotactic radiotherapy QA and dosimetry
}

J H. D. Wong

University of Wollongong, jhdw334@uow.edu.au

T Knittel

Prince of Wales Hopsital

S Downes

Prince of Wales Hospital

M Carolan

University of Wollongong, mcarolan@uow.edu.au

M L. F. Lerch

University of Wollongong, mlerch@uow.edu.au

See next page for additional authors

Follow this and additional works at: https://ro.uow.edu.au/engpapers

Part of the Engineering Commons

https://ro.uow.edu.au/engpapers/3125

\section{Recommended Citation}

Wong, J H. D.; Knittel, T; Downes, S; Carolan, M; Lerch, M L. F.; Petasecca, M; Perevertaylo, V L.; Metcalfe, $\mathrm{P}$; Jackson, M; and Rosenfeld, Anatoly B.: The use of a silicon strip detector dose magnifying glass in stereotactic radiotherapy QA and dosimetry 2011, 1226-1238.

https://ro.uow.edu.au/engpapers/3125

Research Online is the open access institutional repository for the University of Wollongong. For further information contact the UOW Library: research-pubs@uow.edu.au 


\section{Authors}

J H. D. Wong, T Knittel, S Downes, M Carolan, M L. F. Lerch, M Petasecca, V L. Perevertaylo, P Metcalfe, M Jackson, and Anatoly B. Rosenfeld 


\title{
The use of a silicon strip detector dose magnifying glass in stereotactic radiotherapy QA and dosimetry
}

\author{
J. H. D. Wong \\ Centre for Medical Radiation Physics, University of Wollongong. New South Wales 2522, Australia \\ and Faculty of Medicine, University of Malaya, Kuala Lumpur 50603, Malaysia \\ T. Knittel and S. Downes \\ Department of Radiation Oncology, Prince of Wales Hospital, New South Wales 2031, Australia \\ M. Carolan \\ Centre for Medical Radiation Physics, University of Wollongong, New South Wales 2522, Australia \\ and Illawarra Cancer Care Centre, Wollongong Hospital, New South Wales 2500, Australia \\ M. L. F. Lerch and M. Petasecca \\ Centre for Medical Radiation Physics, University of Wollongong, New South Wales 2522, Australia \\ V. L. Perevertaylo \\ SPA-BIT, Kiev, Ukraine 04136 \\ P. Metcalfe \\ Centre for Medical Radiation Physics, University of Wollongong, New South Wales 2522, Australia \\ M. Jackson \\ Department of Radiation Oncology, Prince of Wales Hospital, New South Wales 2031, Australia \\ A. B. Rosenfeld ${ }^{\text {a) }}$ \\ Centre for Medical Radiation Physics, University of Wollongong, New South Wales 2522, Australia
}

(Received 28 June 2010; revised 6 January 2011; accepted for publication 7 January 2011; published 14 February 2011)

Purpose: Stereotactic radiosurgery/therapy (SRS/SRT) is the use of radiation ablation in place of conventional surgical excision to remove or create fibrous tissue in small target volumes. The target of the SRT/SRS treatment is often located in close proximity to critical organs, hence the requirement of high geometric precision including a tight margin on the planning target volume and a sharp dose fall off. One of the major problems with quality assurance (QA) of SRT/SRS is the availability of suitable detectors with the required spatial resolution. The authors present a novel detector that they refer to as the dose magnifying glass (DMG), which has a high spatial resolution $(0.2 \mathrm{~mm})$ and is capable of meeting the stringent requirements of QA and dosimetry in SRS/SRT therapy.

Methods: The DMG is an array of 128 phosphor implanted $n^{+}$strips on a $p$-type Si wafer. The sensitive area defined by a single $n^{+}$strip is $20 \times 2000 \mu \mathrm{m}^{2}$. The Si wafer is $375 \mu \mathrm{m}$ thick. It is mounted on a $0.12 \mathrm{~mm}$ thick Kapton substrate. The authors studied the dose per pulse (dpp) and angular response of the detector in a custom-made SRS phantom. The DMG was used to determine the centers of rotation and positioning errors for the linear accelerator's gantry, couch, and collimator rotations. They also used the DMG to measure the profiles and the total scatter factor $\left(S_{\mathrm{cp}}\right)$ of the SRS cones. Comparisons were made with the EBT2 film and standard $S_{\mathrm{cp}}$ values. The DMG was also used for dosimetric verification of a typical SRS treatment with various noncoplanar fields and arc treatments when applied to the phantom.

Results: The dose per pulse dependency of the DMG was found to be $<5 \%$ for a dpp change of 7:5 times. The angular response of the detector was investigated in the azimuthal and polar directions. The maximum polar angular response was $13.8 \%$ at the gantry angle of $320^{\circ}$, which may be partly due to the phantom geometry. The maximum azimuthal angular response was $15.3 \%$ at gantry angles of $90^{\circ}$ and $270^{\circ}$. The angular response at the gantry angle of $180^{\circ}$ was $6.3 \%$. A correction function was derived to correct for the angular dependence of the detector, which takes into account the contribution of the azimuthal and polar angular response at different treatment couch positions. The maximum positioning errors due to collimator, gantry, and couch rotation were $0.2 \pm 0.1$, $0.4 \pm 0.1$, and $0.4 \pm 0.2 \mathrm{~mm}$, respectively. The $S R S$ cone $S_{\mathrm{cp}}$ agrees very well with the standard data with an average difference of $1.2 \pm 1.1 \%$. Comparison of the relative intensity profiles of the DMG and EBT2 measurements for a simulated SRS treatment shows a maximum difference of $2.5 \%$. 
Conclusions: The DMG was investigated for dose per pulse and angular dependency. Its application to SRS/SRT delivery verification was demonstrated. The DMG with its high spatial resolution and real time capability allows measurement of dose profiles for cone applicators down to $5 \mathrm{~mm}$ in diameter, both accurately and rapidly as required in typical SRS/SRT deliveries. (c) 2011 American Association of Physicists in Medicine. [DOI: 10.1118/1.3549759]

Key words: silicon strip detector, SRS, steep dose gradient, QA, dose magnifying glass (DMG)

\section{INTRODUCTION}

Stereotaxy is a method by which a point is defined within the patient's body by an external three-dimensional coordinate system. ${ }^{1}$ Stereotactic radiosurgery (SRS) is the use of radiation ablation in place of conventional surgical excision to remove or modify a benign lesion in the body. ${ }^{2}$ Traditionally, this method requires a delivery of a large dose in a single treatment, resembling a surgical procedure. In malignancy cases, the treatment is carried out using a series of equal dose fractions, this is then termed stereotactic radiotherapy (SRT). The intention of the SRT/SRS treatment is to deliver a concentrated dose to a small volume of tumor tissue, usually located in close proximity to critical organs. Or, in other cases, it is intended as a boost dose to the target volume. The nature of SRT/SRS treatment requires it to have a very high geometric precision, i.e., a tight margin for the planning target volume (PTV) and a sharp dose fall off. ${ }^{1}$ Due to the high dose delivery and tight margins required in this technique, the planning and delivery of the treatment requires great precision and accuracy.

Existing problems concerning SRT/SRS treatment are twofold; (i) the use of a small field size for treatment and (ii) the associated quality assurance (QA) involved in verifying dose delivery. Due to the exceedingly high dose delivered during a single SRS treatment, this technique is limited to treatment of small lesions, usually though not exclusively to those that are $<4.0 \mathrm{~cm}$ in diameter. ${ }^{3}$ Using small field sizes for radiation delivery raises the question of lateral electronic disequilibrium within the field. This is because the track length of the secondary electron is comparable to or larger than the treatment field size. Lateral electronic disequilibrium usually exists at the field edge where more secondary electrons generated by the photons within the field edge are scattered out of field, while less electrons are scattered inward. With large field sizes, this does not affect the dose at the center of the radiation field. However, with small field sizes, the effect is significant enough to cause a lower total dose at the center of the radiation field. This condition is worsened by the presence of low density inhomogeneous media, such as air cavities and lung, because secondary electrons can travel further in a low density medium. The presence of finite size radiation detectors, in which Bragg-Gray condition is not satisfied, would also cause perturbation to the radiation field. ${ }^{4-7}$

To accurately predict the dose to be delivered, medical physicists must obtain empirical beam data or make Monte Carlo type calculations. Accurate measurements of the dose characteristics (percent depth doses, output factors, and beam profiles) are needed. This information is then entered into a radiotherapy treatment planning system for dose calculation. ${ }^{z}$ Inaccuracies in the beam data will produce modeling errors for the dose distribution and dose calculation errors in the treatment plans. Laub and Wong ${ }^{9}$ reported local discrepancies of $>10 \%$ between calculated cross profiles and profiles measured with films due to insufficient spatial resolution of the detector that was used to collect the beam data during commissioning of the IMRT planning tool. The effect of finite detector size was manifested in the artificial broadening of penumbra width, resulting in a systematic overirradiation of the organ-at-risk arising from attempts to ensure sufficient PTV coverage during planning. ${ }^{8,10}$ The absolute dose difference was reduced to $<2 \%$ simply by using a detector with a smaller cross section, such as $0.015 \mathrm{~cm}^{3}$ pinpoint ion chamber."

According to Pappas et al. ${ }^{8}$ the ideal detector suitable for small field size beam measurements should have the following characteristics: (i) Small sensitive volume to avoid volume averaging effects and the capability of high spatial resolution, (ii) features to overcome the problem of positioning accuracy in small fields, (iii) tissue equivalent and small perturbation of radiation beam, and (iv) show energy, dose rate, and directional response dependence that is consistent and characterizable. To date, there is no detector that can satisfy all of the above criteria. Radiographic film dosimetry has excellent spatial resolution, but is nontissue equivalent, is energy dependent, and is somewhat effected by processing conditions. Radiochromic (Gafchromic) film is almost tissueequivalent, self-developing, and its ease of handling in room light makes this a convenient dosimeter for small field dosimetry. All film methods are nonreal time and are potentially affected by polarization effects and nonuniformities in commercial film scanners. ${ }^{11-13}$ Silicon diodes are able to achieve high spatial resolution with small sensitive volume, but their energy, angular, temperature, and dose rate dependency require rigorous characterization. ${ }^{5,14}$ Diamond detectors are lauded for their near tissue equivalence and small sensitive volume, although one needs to understand their dose rate dependence and significant preirradiation dose effect. ${ }^{3,8} \mathrm{Gel}$ dosimetry has the advantage of having the same medium for dosimetry and dose scattering, water equivalence, and having high spatial resolution in all three dimensions. It is, however, affected by magnetic resonance imaging- and gel-related nonuniformity and artifacts. ${ }^{15}$ In dealing with small field dosimetry, accuracy in positioning of detector relative to the radiation beam becomes an important factor. It is common for SRS treatment to have treatment plans that consist of various noncoplanar deliveries. The uncertainties resulting 
from the collimator, gantry, and couch rotations could certainly influence the beam targeting accuracy with reference to the lesion. In terms of dosimetric verification, positioning accuracy of a small single detector could possibly contribute to a large discrepancy in the measured dose. The common practice is to perform measurements with various detectors as a method to cross check and complement each other.

In this paper, a novel silicon strip detector with a submillimeter spatial resolution was used in SRT/SRS dosimetry. This detector was designed by the Centre for Medical Radiation Physics (CMRP), University of Wollongong and was referred to by Wong et al. If $^{\text {as }}$ "dose magnifying glass" (DMG). We investigated the dose per pulse (dpp) dependency and angular response of the DMG. The effect of packaging was studied, comparing ceramic and Kapton mounting. We measured the SRS cones total scatter factor $\left(S_{c p}\right)$, profiles, and penumbra. Finally, we demonstrated the use of this detector in the determination of the center of rotation and the dosimetric verification of a simulated clinical SRT treatment.

\section{MATERIAL AND METHODS}

\section{II.A. Description of silicon strip detector DMG}

The DMG is an array of 128 phosphor implanted $n^{+}$strips on a $p$-type $S i$ wafer. The sensitive area defined by a single $n^{+}$strip is $20 \times 2000 \mu \mathrm{m}^{2}$. The thickness of the Si wafer is $375 \mu \mathrm{m}$ and the strip pitch is $200 \mu \mathrm{m}$. We produced two types of Si strip detectors based on $p$-Si, one of high resistivity $(5 \mathrm{k} \Omega \mathrm{cm})$ float zone silicon ${ }^{17}$ and the other of low resistivity $(10 \Omega \mathrm{cm})$ Czochralski silicon. ${ }^{17}$ The Si detectors used for the measurements in this work were of the low resistivity type and preirradiated with $1 \mathrm{MeV}$ electrons up to $15 \mathrm{kGy}$.

Silicon detectors are well known for their directional response dependencies that strongly depend on detector's packaging. However, recent publications showed reduced angular response with new diodes. ${ }^{18-21}$ Previous work ${ }^{16}$ on this DMG discovered up to $28 \%$ angular response dependency of the DMG. This was attributed to the design of detector packaging and inherent anisotropy of the Si detector. In terms of packaging, the prototype version of the DMG has a ceramic substrate mounting at the sides and an inhomogeneous encapsulation medium, consisting of solid water and Perspex phantom. In this work, the packaging of the DMG was changed to a $0.12 \mathrm{~mm}$ thick Kapton substrate (Fig. 1). The whole detector with the Kapton was encapsulated in a solid water holder, which was machined to fit snugly with the device, reducing the presence of air gaps between the solid water holder and the detector. The thin Kapton substrate has a density of $1.42 \mathrm{~g} / \mathrm{cm}^{3}$ and has an effective atomic number, $Z_{\text {eff }}=6.6 .^{22}$ This is more tissue equivalent than ceramic $\left(\mathrm{Al}_{2} \mathrm{O}_{3}, Z_{\text {eff }}=11.2\right)$ and is expected to create less perturbation to the radiation beam. It is therefore expected to reduce the angular dependency of the detector due to the detector packaging. The detector array is mounted on the end of a 170 mm long Kapton pigtail. This enables the positioning of the Si detector in phantoms to provide for sufficient scattering.

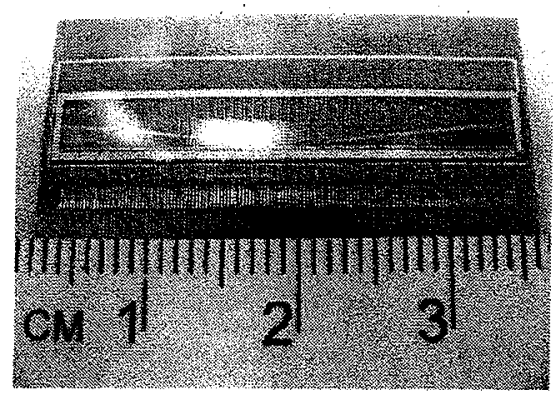

FIG. 1. CMRP DMG is mounted at the end of a $170 \mathrm{~mm}$ Kapton pigtail. The Kapton is $0.12 \mathrm{~mm}$ thick.

The 128 channel readout of the DMG was designed to work with two TERA 64 channel chips. The TERA chip is a Very Large Scale Integration Application Specific Integration Circuit (VLSC ASIC) that was designed by Istituto Nazionale di Fisica Nucleare (INFN)-Torino Division and University of Torino microelectronics group. The ASIC is based on a current to frequency converter, followed by a digital counter. In this way, the charge can be measured by counting the number of pulses coming out from the converter in a given time. The conversion is based on the recycling integrator principle. Each ASIC has 64 independent channels. Readers are referred to Ref. 16 for details of the TERA chip.

\section{II.B. Description of SRS phantom}

A custom-made phantom was designed and machined for measurement of SRS deliveries. It was made of water equivalent material (RW3, Scanditronix Wellhöfer, Germany), ${ }^{2.3}$ which is primarily made up of white polystyrene with $2.1 \%$ titanium oxide $\left(\mathrm{TiO}_{2}\right)$. It was machined into a cylinder with a radius of $90 \mathrm{~mm}$ and a hemispherical end cap. It has a rectangular cavity $\left(100 \times 160 \times 20 \mathrm{~mm}^{3}\right)$ in the middle of the phantom that allows the DMG with its solid water holder ( $50 \mathrm{~mm}$ wide) to fit in accurately together with some solid water spacers for positional adjustability. The cavity width was made wider than the DMG holder so as to allow for $\pm 25 \mathrm{~mm}$ lateral shifting of the DMG within the phantom. The smallest solid water spacers were of $5 \mathrm{~mm}$ width, allowing lateral shifting of the DMG in steps of 5 $\mathrm{mm}$. The center of the sensitive volume of the Si wafer was made pass through the center of the hemispherical component. The SRS phantom has been fitted with an adapter plate which allows it to be bolted onto the Radionics SRS couch mounting frame (Radionics, Inc., Burlington, MA), which, in turn, was attached to the linac couch top (see Fig. 2). The center of the phantom was positioned at the isocenter, at a source to detector distance (SDD) of $100 \mathrm{~cm}$ to allow for accurate, reproducible, and easy setups for DMG SRS measurements.

\section{II.C. Detector relative sensitivity factor measurement}

The 128 channels on the DMG are coupled to individual charge amplifiers whose response may be slightly different 


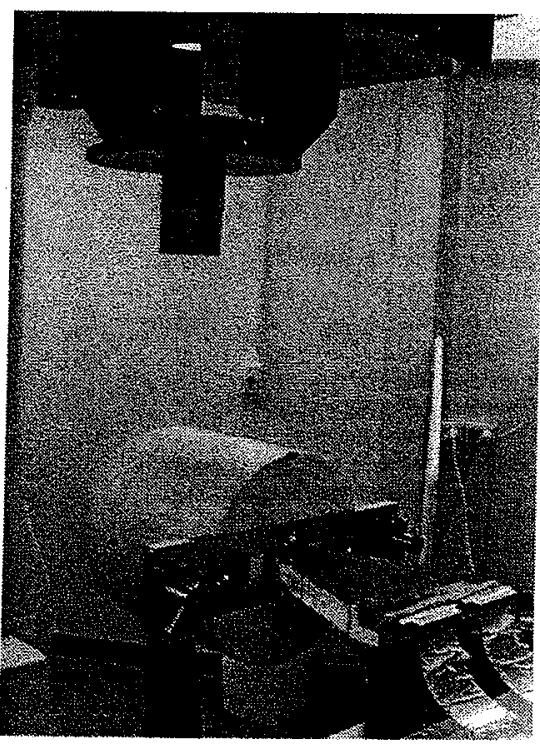

FIG. 2. SRS phantom with the DMG in solid water holder. The SRS phantom is mounted onto the SRS mounting on the linear accelerator couch.

from each other. For all measurements described in this paper, the DMG was used in conjunction with the SRS phantom that has a cylindrical body. A flat calibration phantom was achieved by using a plate with a flat top and matching concave bottom surface over the cylindrical and hemispherical part of the phantom. However, linac beam profiles are not perfectly flat at moderate depths; hence, the method described below was used to generate a sensitivity map of the detector channels. This map was subsequently applied to the other measurements to correct for the detector response.

The detector sensitivity map was derived by employing a shifting and multiple measurements method. The DMG readings, $R_{n i}$, are the product of the detector response, $G_{n i}$, and dose, $D_{n i}$. The index " $n$ " represents the measurement sequence $(1,2$, or 3$)$ and the index " $i$ " represents the detector elements. However, the detector responses, $G_{n i}$, are affected by two factors, the inherent detector sensitivity coefficient, $s_{i}$, and the variation in the linac beam profile, $g_{n i}$, used for uniformity calibration. The two factors need to be separated in order to determine the true detector sensitivity factor,

$$
R_{n i}=G_{n i} \cdot D_{n i}=s_{i} g_{n i} \cdot D_{n i}
$$

A total of three measurements were made. The first measurement was taken with the DMG at isocenter position, while the second and third measurements were taken with the DMG shifted laterally by +5 and $-5 \mathrm{~mm}$ within the phantom cavity.

For any measurement label " $x$," the detector to detector relationships were given initial values. This is achieved by normalizing each detector's response, $R_{x i}$, to the center detector, $R_{x o}$, of the dose profile that is not uniformity corrected. The normalization coefficients, $k_{x i}$, obtained were then applied onto all three measurements,
Normalization coefficient, $k_{x i}=\frac{R_{x i}}{R_{x o}}$.

The next step involves determining the detector response variation due to the linac beam profile. The normalized DMG readings, $R_{n i}^{\prime}$, are the product of detector response purely due to linac dose profile variation, $g_{n i}$, and dose, $D_{n i}$,

$$
\text { Normalized DMG reading, } R_{n i}^{\prime}=\frac{R_{n i}}{k_{x i}} \text {. }
$$

By the shifting and multiple measurement method, the detector response and measured dose at every $5 \mathrm{~mm}$ interval were derived. The dose points obtained were fitted with a spline curve to produce an estimated dose profile, $D$ fit $t_{i}$.

The detector sensitivity coefficient, $s_{i}$, for each detector was derived by dividing the original measurement " $x$ " readings, $R_{x i}$, by $D$ fit $_{i}$. Detector relative sensitivity factors, $f_{i}$, were obtained by taking the ratio of the sensitivity coefficients of each individual channel to the sensitivity coefficient of the center channel,

$$
\text { Detector sensitivity coefficient, } s_{i}=\frac{R_{x i}}{D \mathrm{fit}_{i}},
$$

$$
\text { Detector relative sensitivity factor, } f_{i}=\frac{s_{i}}{s_{o}} \text {. }
$$

\section{II.D. Dose per pulse dependency measurement}

The dose from a linac is delivered in the form of short (microsecond) pulses and linac dose rate is varied by changing the number of pulses per minute. The dose during each pulse is sufficiently high to cause a variation in the silicon detector response. It is therefore important to characterize new detectors for dose per pulse sensitivity. For the dose per pulse measurement, the DMG was mounted on an I' $m$ RT phantom [IBA (Ref. 24)] at SDD of $100 \mathrm{~cm}$ and irradiated with a $10 \times 10 \mathrm{~cm}^{2}$ field beam. The I' $m$ RT phantom was also made of RW3 material. A Varian Clinac 21EX (Varian, Palo Alto, CA) linear accelerator was used to deliver $122 \mathrm{MU}$ (equivalent to $1 \mathrm{~Gy}$ at $\operatorname{SDD} 100 \mathrm{~cm}, 9 \mathrm{~cm}$ depth) with a 6 MV photon beam. To obtain a dose per pulse change, the source to detector distance was varied from 91 to $158 \mathrm{~cm}$, as well as putting various attenuators in the beam path. The lowest dose per pulse was obtained when the DMG was placed under closed solid collimator jaws at $100 \mathrm{~cm}$ SDD. The reference dose at each dose rate was measured with a CC13 ion chamber (Scanditronix Wellhöfer) with a similar setup. The ion recombination test was performed for the CC13 ion chamber according to the TRS-398 protocol and the ion chamber dose rate dependence was measured to be $<0.4 \%$ over the range of dose rates used. The ion chamber data were not corrected for ion recombination. The number of dose pulses delivered was estimated from vendor information, ${ }^{25}$ while the total time required to deliver the 122 MU was obtained from the DMG measurement data. The number of pulses and the dose delivered were then used to 


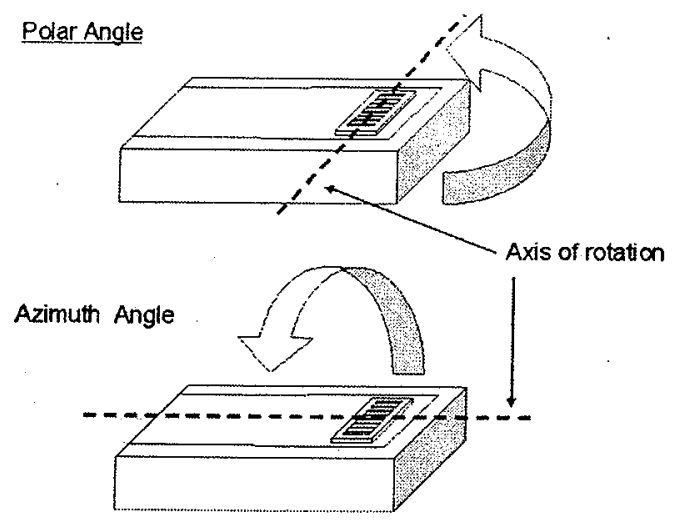

FIG. 3. Diagram showing polar and azimuthal angle.

calculate the dose per pulse measured by the $\mathrm{CC} 13$ and DMG.

\section{II.E. Angular response dependency measurement}

Silicon detectors are known for their directional response dependencies and methods have been devised to reduce the diode angular dependency. ${ }^{16,18-21}$ The large angular response found in the prototype DMG (Ref. 16) was partly attributed to the detector's packaging. The design of the prototype detector with its ceramic mounting at the sides causes a large angular response to the DMG when the beam is parallel to the detector plane. The packaging of the DMG was subsequently improved by mounting the Si wafer on a $0.12 \mathrm{~mm}$ thick Kapton substrate. In this study, we investigated the angular response of the DMG in two orthogonal directions, i.e., the azimuth angles and polar angles. Refer to Fig. 3 for pictorial definition of polar and azimuth angles.

For the angular response measurements, the DMG was positioned at SDD $100 \mathrm{~cm}, 9 \mathrm{~cm}$ depth of the SRS phantom. For azimuth angle measurements, the couch angle was set to $0^{\circ}$ and a small field size of $2.5 \times 2.5 \mathrm{~cm}^{2}$ was used. The gantry was rotated from $0^{\circ}$ to $350^{\circ}$ with a stepping angle of $10^{\circ}$ intervals and $100 \mathrm{MUs}$ were delivered at each angle.

For measurement of polar angles, the couch was rotated $270^{\circ}$. Due to the design of the detector mounting on one end of the Kapton pigtail and the logistics of the readout connection/cables at the other end of the Kapton pigtail, polar angle measurements were limited to gantry angles of $320^{\circ}-180^{\circ}$, clockwise. Measurements were taken in $10^{\circ}$ increments, with a $2.5 \times 2.5 \mathrm{~cm}^{2}$ radiation field size.

The DMG detector response was taken as the average response of the $S i$ strips located within $\pm 1 \mathrm{~mm}$ of the axis of rotation. The angular dependence of the DMG was taken as a ratio of the DMG response at different angles to the DMG response when the radiation beam was perpendicular to the detector plane, corresponding to the gantry angle of $0^{\circ}$,
Angular dependence, $A_{\theta}=\frac{R_{\theta}}{R_{\theta}}$,

where $R_{\theta}$ is the response at gantry angle $\theta^{\circ}$ and $R_{0}$ is the response at the gantry angle of $0^{\circ}$.

The angular dependence is defined separately for the polar and azimuthal angles. The angular correction factor $\left(\mathrm{CF}_{p}\right.$ and $\mathrm{CF}_{a}$ ) is the inverse of the angular dependence,

$$
\text { Polar angle correction factor, } \mathrm{CF}_{p}=\frac{1}{A_{\theta p}}
$$

and

$$
\text { Azimuth angle correction factor, } \mathrm{CF}_{a}=\frac{1}{A_{\theta a}} \text {. }
$$

However, the final angular response dependency was derived from the combined contribution of the azimuthal and polar angular response. This was then used to correct for the data measured in the SRS dosimetric plan verification.

It is common for any SRS delivery to incorporate various noncoplanar deliveries. This is essential to produce a highly focused irradiation on the lesion while reducing doses to normal tissue by spreading it to larger volumes and to avoid critical structures. A noncoplanar plan would involve many couch angles. This means that the radiation beam could reach the detector from many polar or azimuthal angles. Hence, the angular correction should take into account the contribution of the polar and azimuth angles based on the position of the couch. The angular correction factors are derived as

$$
\begin{aligned}
& \mathrm{CF}_{\text {angle }}(\varphi, \theta) \\
& \quad=\sqrt{\left(\sin (\varphi) \cdot \mathrm{CF}_{p}(\theta)\right)^{2}+\left(\cos (\varphi) \cdot \mathrm{CF}_{a}(\theta)\right)^{2}} \text { for } 0^{\circ} \\
& \quad \leq \varphi \leq 90^{\circ}
\end{aligned}
$$

and

$$
\begin{aligned}
\mathrm{CF}_{\text {angle }}(\varphi, \theta) & \\
= & \sqrt{\left(\sin (360-\varphi): \mathrm{CF}_{p}(\theta)\right)^{2}+\left(\cos (360-\varphi) \cdot \mathrm{CF}_{a}(\theta)\right)^{2}} \\
& \text { for } 270^{\circ} \leq \varphi<360^{\circ},
\end{aligned}
$$

where $\varphi$ is the couch angle and $\theta$ is the gantry angle.

\section{II.F. Determination of center of rotation (COR) and positioning error}

Positioning uncertainty in small field setups using small single element detectors is one of the challenges involved in the dosimetric verification of SRS treatments. The collimator, gantry, and couch rotations involved in SRS delivery produce an uncertainty in beam targeting and dosimetric verification. Understanding this positioning variability would allow us to evaluate the uncertainty involved in SRS dosimetry:

The COR of the linac collimator was determined from the center of the beam profiles obtained from the $0^{\circ}$ and $180^{\circ}$ collimator rotation angles. The center of the profiles was 
obtained from the midpoint between the $50 \%$ of the intensity profiles. The CORs of the linac gantry and couch were determined likewise

$$
\text { Center of rotation, } x_{\text {cor }}=\frac{x_{1}+x_{2}}{2} \text {, }
$$

Positioning error, $x_{\text {err }}=\frac{x_{1}-x_{2}}{2}$,

where $x_{1}$ and $x_{2}$ are the midpoint coordinates between the $50 \%$ coordinates of the intensity profiles

\section{II.G. SRS cone profiles and total scatter factor $\left(S_{c p}\right)$ measurement}

The profiles and $S_{\mathrm{cp}}$ for the SRS cones with diameter ranging from 5 to $40 \mathrm{~mm}$ were measured. The DMG was positioned at SDD of $100 \mathrm{~cm}$. The measurements were compared to Gafchromic EBT2 (ISP, Wayne, NJ) film measurements and the standard data. The standard data were measured with a CCO4 ion chamber (Scanditronix Wellhöfer) and verified with Monte Carlo calculation. The $\mathrm{CCO} 4$ ion chamber was used to measure the $S_{\mathrm{cp}}$ for the cone diameters of $15-40 \mathrm{~mm}$ only. Monte Carlo calculations were made with voxel size of $1 \times 1 \times 2 \mathrm{~mm}^{3}$ for cone diameter $\leq 10 \mathrm{~mm}$ and voxel size of $2 \times 2 \times 2 \mathrm{~mm}^{3}$ for cones with diameter of $12.5-40 \mathrm{~mm}$. The $S_{\mathrm{cp}}$ values based on the measurements were rescaled to match the Monte Carlo data for the $15 \mathrm{~mm}$ diameter cone. For the film measurements, a specially made film insert was made to fit inside the SRS phantom cavity. The film insert had a small slit to fit a piece of $40 \times 100 \mathrm{~mm}^{2}$ EBT2 film. It was made from the same RW3 material as the rest of the SRS phantom.

The films were scanned after $24 \mathrm{~h}$ to allow for stabilization of the postirradiation coloration. An A3 size flatbed scanner (Epson Expression 10000XL) was used. Each film was scanned six times. Only the last three scans were kept for image analysis. This was to ensure that the scanner was sufficiently warmed up and thus ensuring consistency in the scanned films. ${ }^{26}$ The films were scanned in 48-bit RGB color with scanning resolution of $150 \mathrm{dpi}$. Care was taken to scan the film in the same orientation at the center region of the scanner to reduce scanner induced nonuniformity ${ }^{12} \mathrm{~A}$ set of calibration films was also irradiated in the same experimental session. Analysis of the images was done using IMAGEJ version 1.39U (National Institutes of Health, USA) software. The red channel of the last three scans for each film was averaged and used for our analysis. ${ }^{27}$ For the $S_{\mathrm{cp}}$ measurement, dose measured by the EBT2 film was averaged over an area of $0.5 \times 2 \mathrm{~mm}^{2}$ (corresponding to $3 \times 12$ pixels) at the center of the beam to match the sensitive area of the DMG.

\section{II.H. Clinical stereotactic arc measurement}

A four arc noncoplanar simulated SRS treatment was carried out with a Siemens Oncor linear accelerator. A $180^{\circ}$ gantry arc angle was used, starting at $0^{\circ}$ and stopping at $180^{\circ}$. The couch angles used were from $270^{\circ}$ to $360^{\circ}$ at $30^{\circ}$

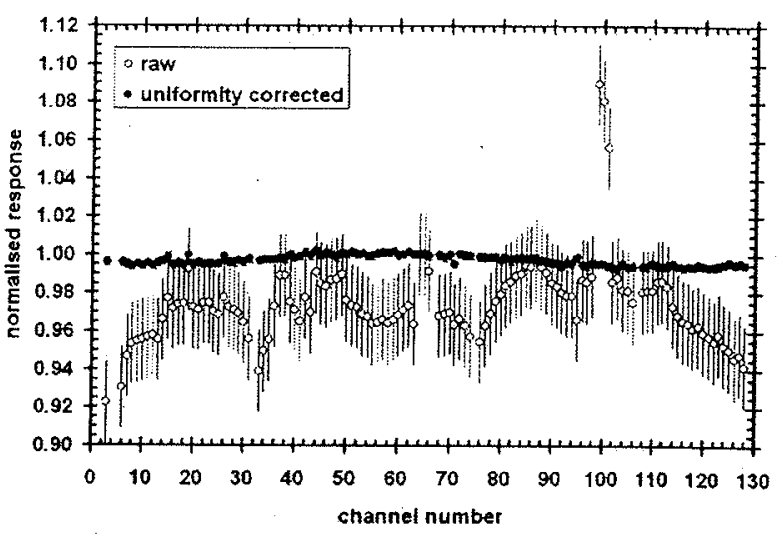

F1G. 4. Detector response of the DMG before and after uniformity correction. The data are normalized to $I$ at the center detector. The error bars represent 1 s.d. of the mean. The $\mathrm{CV}$ before correction was $>2 \%$. After uniformity correction, the $C V$ was less than $0.5 \%$.

intervals. The center of the DMG was positioned at the isocenter. The whole delivery was carried out using an $8 \mathrm{~mm}$ diameter cone. For each arc, $100 \mathrm{MU}$ was delivered. These measurements were compared to Gafchromic EBT2 films using the same protocol.

\section{RESULTS}

\section{III.A. Uniformity}

Figure 4 shows the detector response before and after the uniformity correction. The data are normalized to the center detector. Error bars represent 1 s.d. of the mean of all the detectors. The coefficient of variation (CV) before uniformity correction was $>2 \%$ and less than $0.5 \%$ after. These sensitivity factors were applied to all subsequent measurements.

\section{III.B. Dose per pulse dependency}

The number of dose pulses delivered was calculated from the information in the linac manual. The Varian Clinac 21EX linear accelerator has a pulse repetition frequency of $360 \mathrm{~Hz}$ for $6 \mathrm{MV}$ photon energy. From the known dose rate, we estimated the dose per pulse as $2.28 \times 10^{-4} \mathrm{~Gy} /$ pulse at source to detector distance of $100 \mathrm{~cm}$. The dose per pulse response of the DMG was taken as the ratio of the measured readings to the ion chamber readings. Figure 5 shows the dose per pulse response of the DMG normalized to 1 at $2.28 \times 10^{-4} \mathrm{~Gy} /$ pulse. For a change in dose per pulse of a factor of 7.5 (corresponding to $3.70 \times 10^{-5}-2.74$ $\times 10^{-4} \mathrm{~Gy} /$ pulse), the change in dose per pulse response is less than 5\%. For a factor of 22 (corresponding to 1.26 $\times 10^{-5}-2.74 \times 10^{-4} \mathrm{~Gy} /$ pulse), the change in dose per pulse response is less than $11 \%$. The DMG under-responded by $50 \%$ compared to $\mathrm{CC} 13$ when measured under closed solid collimator jaws (corresponding to $1.15 \times 10^{-6} \mathrm{~Gy} /$ pulse), where the dose per pulse decreased by 238 -fold relative to the highest measured value of $2.74 \times 10^{-4} \mathrm{~Gy} /$ pulse. This only affects the measurements when the radiation is highly 


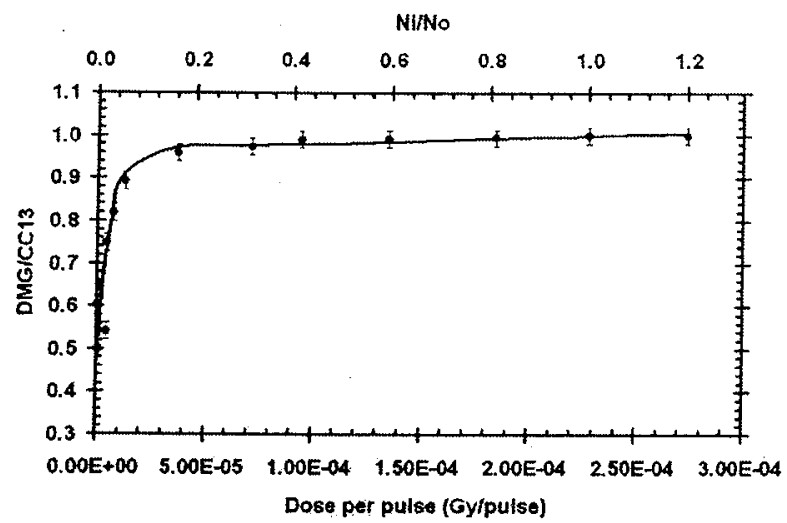

FIG. 5. Dose per pulse dependency of DMG taken as the measured DMG counts over the $\mathrm{CCl} 3$ readings. The measured data are normalized to I at dose per pulse of $2.28 \times 10^{-4}$, corresponding to SDD of $100 \mathrm{~cm}$. The error bars represent the reproducibility of the measurements. The data points were curve fitted with a double exponential function, with $r^{2}$ of 0.96 . The bottom axis shows the dose per pulse in units of Gy/pulse. The top axis shows the $N_{i} / N_{o}$ relationship.

attenuated. Compared to the dpp measured in Ref. 16, the dpp measured in this version of the DMG appears to have stronger dpp dependence. One of the reasons may be due to the difference in the detector used. The detector used in this work is different in its sensitive volume and preirradiation condition to the one used in Ref. 16.

A correction function was derived to correct for the dose per pulse dependence. From Fig. 5 , the data were curve fitted with a double exponential function giving a goodness of fit $r^{2}=0.96$. The dose per pulse effect $(\alpha)$ is an exponential function of the measured signal $\left(N_{i}\right)$ to signal at 2.28 $\times 10^{-4} \mathrm{~Gy} /$ pulse $\left(N_{o}\right)$ ratio,

Dose per pulse effect, $\alpha=f(x)=a e^{(b x)}+c e^{(d x)}$,

where $x=N_{i} / N_{o}$.

The corrected signal $N_{c}$ is given by

$$
N_{c}=N_{i} \times\left(\frac{1}{\alpha}\right)_{N_{i} / N_{o}} \text {. }
$$

\section{III.C. Angular response dependency}

Figure 6 shows the polar and azimuth angular dependency of the DMG. The angular response was defined as the mean value of all channels within $1 \mathrm{~mm}$ from the center of the detector. The maximum polar angular response was $13.8 \% \pm 0.1 \%$ at a gantry angle of $320^{\circ}$ (at couch position $270^{\circ}$ ). If the couch position is at $90^{\circ}$, the profile of the polar angular response would be mirrored on the graph's $0^{\circ}-180^{\circ}$ axis. The apparent under-response in polar angular response at gantry angles of $320^{\circ}-350^{\circ}$ may be due to the cylindrical portion of the phantom rather than the intrinsic detector response. The maximum azimuthal angular response was at gantry angles of $90^{\circ}$ and $270^{\circ}$, with an angular response deviation from unity of $15.3 \% \pm 0.1 \%$. Compared to the prototype version, ${ }^{16}$ the maximum azimuthal angular response

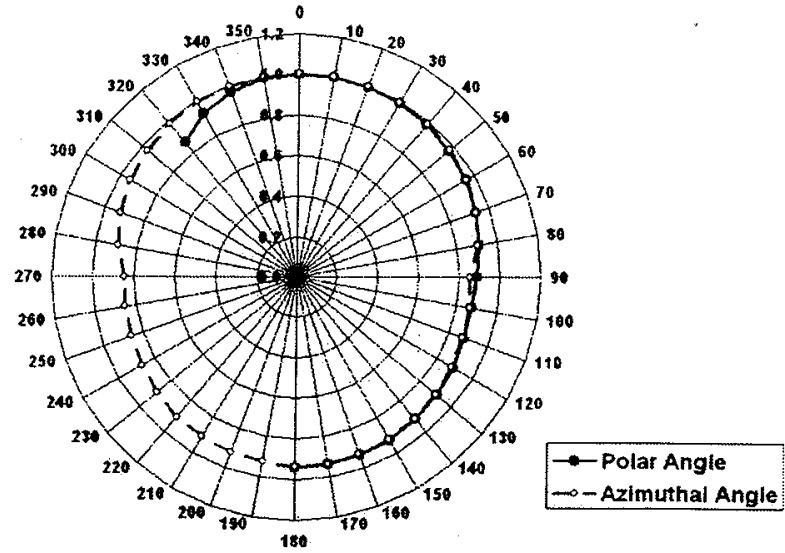

FiG. 6. Polar and azimuthal angular dependence of DMG in the SRS phantom. The angles refer to the linear accelerator gantry angles. The angular response is normalized to $l$ at gantry angle of $0^{\circ}$. The polar angular response is for the couch position of $270^{\circ}$.

of the DMG mounted on $0.12 \mathrm{~mm}$ of Kapton substrate was reduced by $12.8 \%$. The polar and azimuthal angular response deviation from unity at gantry angle $180^{\circ}$ was $6.3 \% \pm 0.2 \%$. The resulting angular response of the DMG is due to the inherent anisotropy of the Si slab/detector.

\section{III.D. COR and positioning error determination}

The center of rotation and positioning error was determined by varying one of the delivery angles (collimator, gantry, or couch) while keeping the other two fixed. The maximum positioning errors due to collimator, gantry, and couch rotation were $0.2 \pm 0.1,0.4 \pm 0.1$, and $0.4 \pm 0.2 \mathrm{~mm}$ ( 1 s.d.), respectively. Figure 7 shows the profiles of two acquisitions within the same isocentric setup but with different couch positions. The data were normalized to unity at the peak of the acquisition for couch position at $90^{\circ}$. The differences in the peaks demonstrate the variability of the positioning of the detector relative to the beam. This also resulted in a higher dose measured in the acquisition for the couch position at $270^{\circ}$. In addition to the measured positioning error due to the

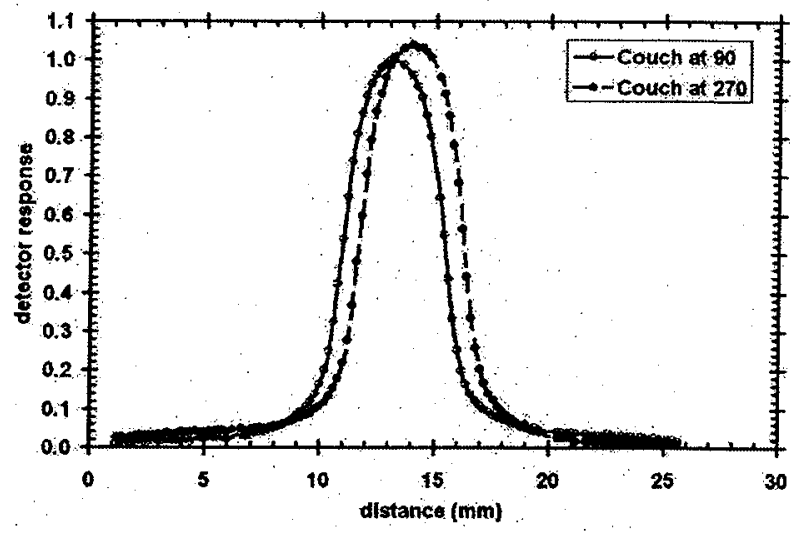

Fig. 7. Two profiles of $5 \mathrm{~mm}$ diameter cones measured with DMG showing discrepancy at the center of rotation due to couch rotation from $90^{\circ}$ to $270^{\circ}$. 


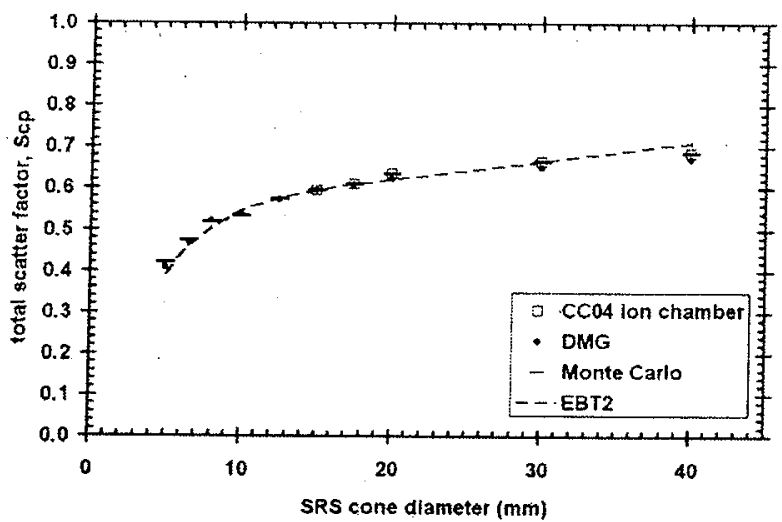

FiG. 8. SRS cone total scatter factor comparing DMG with EBT2, CCO4 measurements, and Monte Carlo calculation. The measurements were taken within the spatial distance of $\pm 0.2 \mathrm{~mm}$ from the center axis. The $\mathrm{CCO} 4$ ion chamber was used to measured $S_{c p}$ for the cones with $15-40 \mathrm{~mm}$. The Monte Carlo calculation was made with voxel size of $1 \times 1 \times 2 \mathrm{~mm}^{3}$ for cone diameter $\leq 10 \mathrm{~mm}$, while voxel size of $2 \times 2 \times 2 \mathrm{~mm}^{3}$ was used to calculate for the cone diameter $>10 \mathrm{~cm}$.

rotating component of the linear accelerator, there is also the uncertainty in the cone positioning in the applicator of $<0.5 \mathrm{~mm}$. With that taken into consideration, the positioning uncertainty added in quadrature was $0.8 \mathrm{~mm}$ for the DMG. This represents the maximum uncertainty of $<2 \%$ in the maximum dose measured at the smallest cone diameter of $5 \mathrm{~mm}$. This test is somewhat similar to the Winston Lutz test $^{28}$ that was used to measure the central axis offset due to the rotating component of the linear accelerator. The DMG, like film, is a useful tool for determination of COR and has the advantage of obtaining immediate profiles and is especially advantageous for the purpose of mechanical alignment of the SRS cones.

Once the positioning error was determined as described above, the calculated positioning error was also applied to the EBT2 film measurements to estimate the uncertainty in the measured dose. However, in addition to the $0.8 \mathrm{~mm}$ positioning error, the use of the film also involves additional positioning uncertainty of $\pm 0.5 \mathrm{~mm}$ when it is placed in the phantom. The positioning uncertainty in the EBT2 is therefore $0.9 \mathrm{~mm}$ and this translates to $\pm 3 \%$ dose uncertainty at maximum dose for $5 \mathrm{~mm}$ diameter cone.

\section{III.E. SRS cones $S_{c p}$ measurement}

The $S_{\mathrm{cp}}$ of the cones were measured with the DMG (Fig. 8) for cone diameters from 5 to $40 \mathrm{~mm}$. The measurements were taken as an average of the two central detectors (sensitive area of $0.4 \times 2.0 \mathrm{~mm}^{2}$ ) of DMG. The results were compared to the EBT2 measurements (averaged over an area of $0.5 \times 2 \mathrm{~mm}^{2}$ ) and the standard data that are based on $\mathrm{CC} 04$ ion chamber measurements for SRS cones with diameter $>10 \mathrm{~mm}$ and Monte Carlo simulations for cones with diameter $\leq 10 \mathrm{~mm}$. The DMG data are rescaled to the calibrated $15 \mathrm{~mm}$ diameter cone of the Monte Carlo data. The EBT2 data were rescaled to the $15 \mathrm{~mm}$ diameter cone value using a fitted curve to avoid the effect of scatter in the data. The
DMG measurements agreed well with the standard data measurements. The average difference between the DMG measurements with the standard data and EBT2 measurements were found to be $1.2 \pm 1.1 \%$ and $1.9 \pm 1.9 \%$, respectively.

The good agreement between the Monte Carlo calculation and the DMG measurements for the $S_{\mathrm{cp}}$ is not unexpected since at the center of the beam, the profile gradient does not change drastically. Volume averaging present at this point of measurement between a detector with the area of $0.02 \times 2$ and $1 \times 1 \mathrm{~mm}^{2}$ would not be significantly different. However, if the same detector was used to measure the high gradient penumbra region of a beam profile with radial symmetry, detector with a $0.02 \times 2 \mathrm{~mm}^{2}$ area may be better due to the $0.02 \mathrm{~mm}$ width of the detector than a $1 \times 1 \mathrm{~mm}^{2}$ area. In this case, averaging effect in the longitudinal direction is not as critical as in the radial direction and averaging effect of the detector is dominated by the size in the radial direction. This means that a detector with an area of $1 \times 1 \mathrm{~mm}^{2}$ will have good agreement with a detector with an area of 0.4 $\times 2 \mathrm{~mm}^{2}$ in $S_{\mathrm{cp}}$ measurements but the detector with an area of $0.02 \times 2 \mathrm{~mm}^{2}$ (DMG) will provide much more realistic beam profile than the detector with an area of $1 \times 1 \mathrm{~mm}^{2}$ and should be in a better agreement with a EBT2 film readout with $0.17 \mathrm{~mm}$ resolution. This point will be elaborated further in Sec. IV.

\section{III.F. SRS cone profiles and penumbra}

The DMG with its array of 128 channels has a sensitive length of $25.6 \mathrm{~mm}$. This allowed us to measure the dose profiles of the small SRS cones with diameters up to $20 \mathrm{~mm}$ without moving the detector. We also measured the $80 \%-$ $20 \%$ penumbra width and the full width at half maximum (FWHM) of the cones. The results were tabulated in Table I. The average differences between the EBT2 and DMG measurements are $0.22 \pm 0.07$ and $0.12 \pm 0.09 \mathrm{~mm}$ for penumbra width and FWHM, respectively.

\section{III.G. Clinical SRS application}

Four SRS arcs spanning $180^{\circ}$ were delivered with couch angles from $270^{\circ}$ to $360^{\circ}$ at every $30^{\circ}$ intervals. The relative intensity profile of the sum of the arcs is shown in Fig. 9. The relative intensity profiles were normalized to 1 at the maximum of the EBT2 measurement and error bars are the $\pm 3 \%$ and $\pm 2 \%$ dose uncertainties in the EBT2 and DMG, respectively. DMG measurements were all corrected for angular dependencies. Within the $\pm 1 \mathrm{~mm}$ positional and $3 \%$ dose uncertainty in the measurement, the EBT2 and DMG agree well with each other. The average difference above the $90 \%$ intensity was $1.7 \pm 0.8 \%$.

\section{DISCUSSION}

The averaging effect of the detector should be considered as at least a two dimensional problem and for the particular field and configuration of the detector. For example, in the case of a SRS radiation field with radial symmetry, different restrictions would apply to the detector geometry for small 
TABLE: I. The penumbra and FWHM measurement of the dose profiles of $5 \mathrm{~mm}$ cone to $20 \mathrm{~mm}$ cone diameter at SDD of $100 \mathrm{~cm}$, comparing DMG and EBT2 measurements.

\begin{tabular}{cccccccc}
\hline & \multicolumn{3}{c}{$\begin{array}{c}\text { Penumbra }(P 80 \%-20 \%) \\
(\mathrm{mm})\end{array}$} & & \multicolumn{3}{c}{$\begin{array}{c}\text { FWHM } \\
(\mathrm{mm})\end{array}$} \\
\cline { 2 - 3 } $\begin{array}{c}\text { Cone diameter } \\
(\mathrm{mm})\end{array}$ & DMG & EBT2 & Difference & DMG & EBT2 & Difference \\
\hline 5.0 & 1.28 & 1.49 & 0.21 & & 4.61 & 4.86 & 0.25 \\
6.5 & 1.41 & 1.60 & 0.19 & & 6.23 & 6.36 & 0.13 \\
8.0 & 1.55 & 1.86 & 0.31 & & 8.24 & 8.32 & 0.08 \\
10.0 & 1.65 & 1.97 & 0.32 & & 9.26 & 9.40 & 0.14 \\
12.5 & 1.91 & 2.11 & 0.19 & 12.00 & 11.82 & 0.18 \\
15.0 & 2.05 & 2.23 & 0.18 & & 14.43 & 14.43 & 0.00 \\
17.5 & 2.12 & 2.24 & 0.12 & 16.50 & 16.52 & 0.02 \\
20.0 & 2.18 & 2.44 & 0.25 & 19.12 & 18.93 & 0.19 \\
\hline \hline
\end{tabular}

beam measurements. The following workings set out the consideration of the effect of volume averaging of detectors at (a) the center of the beam (such as $S_{\mathrm{cp}}$ measurement) and (b) at the penumbra region. The volume averaging effect of detectors with different geometrical configurations was estimated analytically (Sec. IV A). The analytical model used was based on the real data measured with EBT2 film (scanned with 150 dpi resolution) [Fig. 10(a)]. Section IV B provides detail of the penumbra consideration.

Consider:

(1) FWHM of beam $=5 \mathrm{~mm}$.

(2) The radiation beam considered is a circular beam with radial symmetry, i.e., the penumbra drop off would be almost symmetrical in every direction, as seen in Fig. $10(\mathrm{~b})$.

(3) Detector area considered $=2 a \times 2 b . \mathrm{mm}$ (where $a=0.2$, $b=1$ for DMG and $a=b=0.5$ for Monte Carlo virtual detector), as seen in Fig. 11 .

\section{IV.A. Center of beam consideration (for $S_{c p}$ measurement)}

The response of detector is $R=\int_{\theta} \int_{r} D(r) r d r d \theta$,

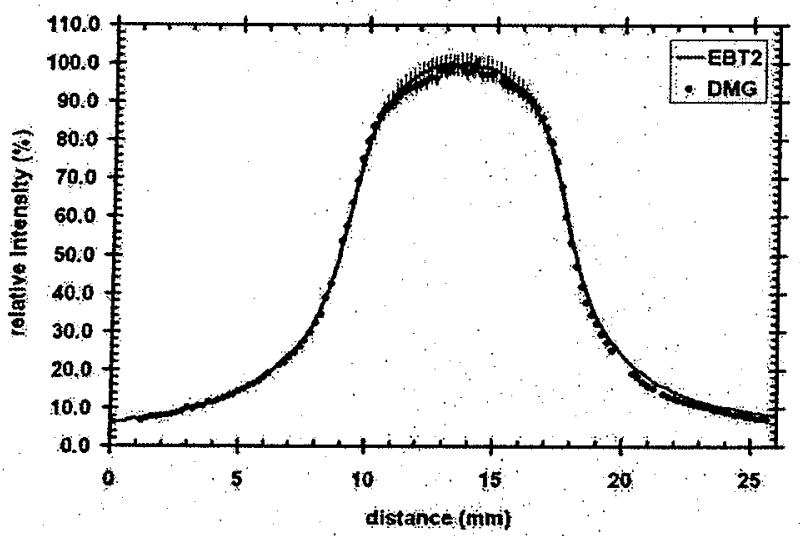

FIG. 9. SRS arc $\left(0^{\circ}-180^{\circ}\right)$ relative intensity profile
To simplify the calculation, only the first quarter of the detector, which is represented by the areas $\mathrm{A} 1$ and $\mathrm{A} 2$, is considered (see Fig. 11),

$$
\begin{aligned}
& R=R_{1}+R_{2}, \\
& R_{1}=\int_{0}^{\theta 1} \int_{0}^{a / \cos \theta} D(r) r d r d \theta, \\
& R_{2}=\int_{\theta 1}^{\pi / 2} \int_{0}^{b / \sin \theta} D(r) r d r d \theta,
\end{aligned}
$$

where $R_{1}$ and $R_{2}$ represent the detector response corresponding to the detector areas $A_{1}$ and $A_{2}$.

For a uniform field with a point dose $D_{o}$, the response of the detector is

$$
R=D_{a} \times a b .
$$

From the film data [Fig. 10(a)], the dose profile was plotted across the center of the beam [Fig. 10(c)]. Since the detector area considered is smaller than the FWHM of the beam profile, we will only consider the central $\pm 2.5 \mathrm{~mm}$ of the profile.

In Fig. $10($ d), the data points $>0.3$ were extracted and plotted as a function of the radial distance, $r$ (mm). The data points were curve fitted with a fourth degree polynomial function $\left(r^{2}=0.998\right)$ using MATLAB. This is the dose function, $D(r)$, that will be used for the following calculation.

Therefore,

$$
\begin{aligned}
R= & \int_{0}^{\theta 1} \int_{0}^{a / \cos \theta}\left(k r^{4}+l r^{3}+m r^{2}+n r+p\right) r d r d \theta \\
& +\int_{\theta 1}^{\pi / 2} \int_{0}^{b / \sin \theta}\left(k r^{4}+l r^{3}+m r^{2}+n r+p\right) r d r d \theta
\end{aligned}
$$

where $\theta 1=\tan ^{-1}(b / a)$ and coefficients $k, l, m n$, and $p$ are generated from MATLAB curve fitting toolbox,

$$
\text { Dose, } D=\frac{R}{a b}
$$

Results on dose averaging effect are shown in Table II. 

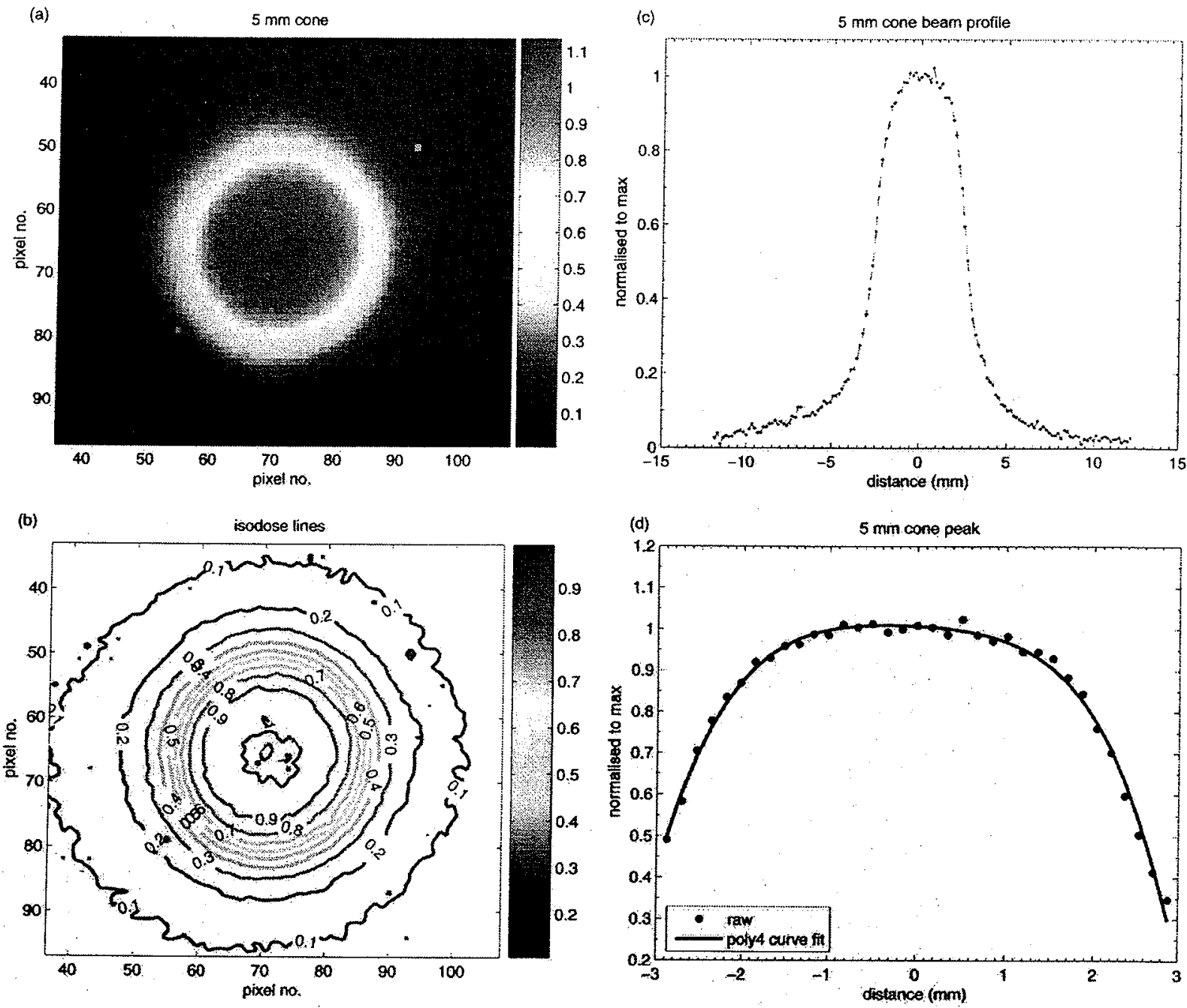

Fig. 10. (a) Pseudocolored image from EBT2 film measurement of $5 \mathrm{~mm}$ diameter beam, normalized to the center of the beam, (b) isodose lines for the $5 \mathrm{~mm}$ diameter beam, (c) signal profile along a horizontal line across the middle of the circular beam, and (d) zoom in to the top of the beam profile, data points $>0.3$ were extracted and curve fitted with a fourth degree polynomial function $\left(r^{2}=0.998\right)$.

This exercise showed that at the center of beam region of a $5 \mathrm{~mm}$ diameter beam, the variation in dose measured by different detectors with dimensions of $1 \times 1$ and 0.4 $\times 2 \mathrm{~mm}^{2}$ is on the order of $0.6 \%$. That is why the Monte Carlo calculation and DMG measurements are in close agreement. The relative dose averaging effect will be the same for the whole detector as for a quarter of the detector considered.

\section{IV.B. Penumbra consideration for beam with radial symmetry}

For the volume averaging effect of detectors at the penumbra region, the hypothesis is that in a radially symmetrical beam, for a detector with dimension of $0.02 \times 2 \mathrm{~mm}^{2}$, the dose gradient in the $r$-direction is steeper than in the $\tau$-direction. Therefore, for penumbra measurement, a small detector width of $0.02 \mathrm{~mm}$ (almost identical to point dose) would be able to represent the real penumbra profile in the $r$-direction. In the $\tau$-direction, on the other hand, although there would be some volume averaging, the effect would be less critical due to the shallower dose gradient. Two methods were used to demonstrate this effect, (i) dose gradient variation in the orthogonal radial direction corresponding to the length of the detector length of interest and (ii) numerical integration of the dose map using areas of $0.2 \times 1$ and 1 $\times 1 \mathrm{~mm}^{2}$.

\section{IV.B.1. Dose gradient variation in the orthogonal radial directions}

Figure 12 shows the schematic diagram of the beam profile from the top view and the denotation of the $r$ - and $\tau$-directions. Two detectors with different longitudinal dimensions of 1 and $2 \mathrm{~mm}$ are considered. However, the dose gradient is only calculated for one-half of the detector length, $b$, 


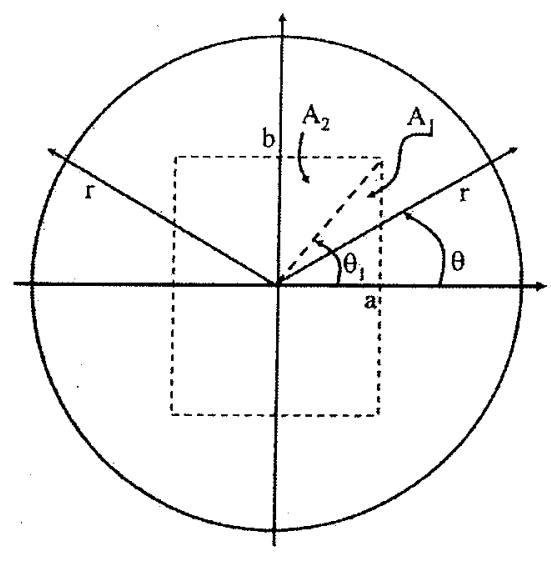

Fig. 11. Schematic drawing of the detector area superimposed on the radial beam contour.

Dose gradient in the $r$-direction $=\frac{d D}{d r}$,

Dose gradient in the $\tau$-direction $=\frac{\Delta D}{b}=\frac{\left(D_{n}-D_{n-1}\right)}{b}$,

where $b=\frac{1}{2}$ of the longitudinal length of the detector.

Figure 13 shows the dose gradient along the $r$-direction (a) and the $r$-direction (b). The dose gradient is much larger in the $r$-direction compared to the $\tau$-direction, particularly at the middle of the penumbra $(r=-2.5 \mathrm{~mm})$, where the dose gradient in the $r$-direction is five times greater than the dose gradient in the $\tau$-direction. It is important to note that the dose gradient in the $\tau$-direction is almost independent on the $b$-size of the detector. A detector with a smaller width, 0.2 $\mathrm{mm}$, would be able to measure the penumbra width more accurately, compared to a $1 \times 1 \mathrm{~mm}^{2}$ detector (e.g., face on stereotactic diode), even though the length of the detector is $2 \mathrm{~mm}$ (e.g., DMG). This is due to the fact that the dose gradient variation in the $\tau$-direction is not significantly different between the two detectors, while the dose gradient in the $r$-direction varies more dramatically. It was also confirmed using numerical simulations (Sec. IV B 2) based on the dose map obtained by the EBT2 film.

\section{IV.B.2. Numerical integration of dose}

From Fig. 10(b), two strips of dose map with areas of 2 $\times 10$ and $1 \times 10 \mathrm{~mm}^{2}$ were extracted (Fig. 14). For the 2

TABLE II. Dose calculated based on the analytical model showing the effect of dose averaging effect with different detector sizes.

\begin{tabular}{ll}
\hline & Dose, $D$ \\
\hline MC $\left(0.5 \times 0.5 \mathrm{~mm}^{2}\right)$ & 0.9993 \\
DMG $\left(0.2 \times 1 \mathrm{~mm}^{2}\right)$ & 0.9931 \\
Difference $(\mathrm{MC}-\mathrm{DMG})$ & 0.0062 \\
\hline
\end{tabular}

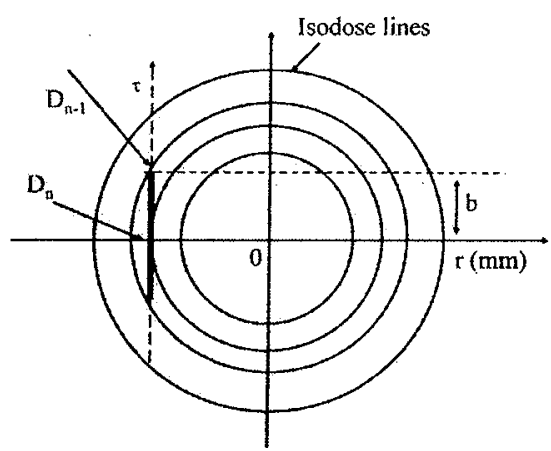

FIG. 12. Schematic diagram representing the top view of the beam and the denotation of the $r$ - and the $\tau$-directions. The isodose lines are represented as the concentric circles.

$\times 10 \mathrm{~mm}^{2}$ strip, the dose was integrated over an area of 2 $\times 0.2 \mathrm{~mm}^{2}$ at $0.2 \mathrm{~mm}$ interval, while for the $1 \times 10 \mathrm{~mm}^{2}$ strip, the dose was integrated over an area of $1 \times 1 \mathrm{~mm}^{2}$ at 1 mm interval. The result of the numerical integration is shown in Fig. 15.

Figure 15 shows that the effect of volume averaging of $0.2 \times 2$ and $1 \times 1 \mathrm{~mm}^{2}$ detectors. At the center of the beam
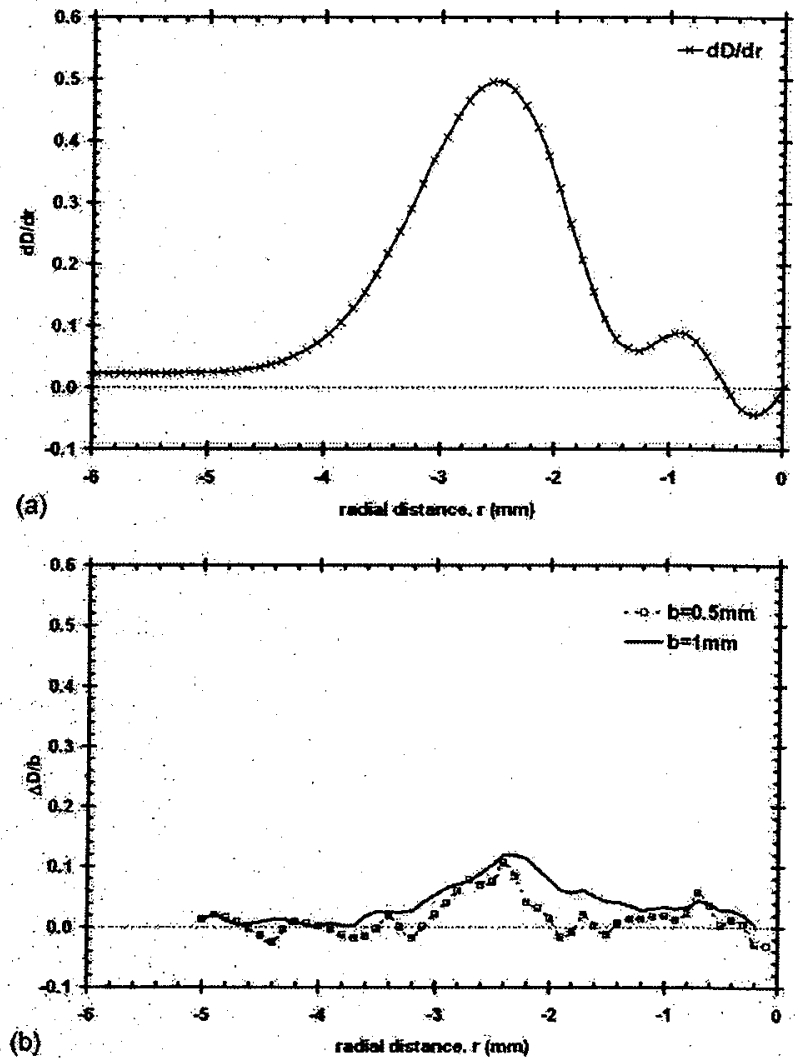

FIG. 13. Dose gradient in (a) $r$ - and (b) $\tau$-directions for detector with $\frac{1}{2}$ longitudinal length of $b=0,5 \mathrm{~mm}$ and $1 \mathrm{~mm}$. The $x$ axis is the radial direction extending from the center of the beam $(r=0 \mathrm{~mm})$ to the edge of the beam $(r=-6 \mathrm{~mm})$. The negative gradient at the region $r=-0.5-0 \mathrm{~mm}$ is due to the noise in the EBT2 film picked up by the fitted curve. 


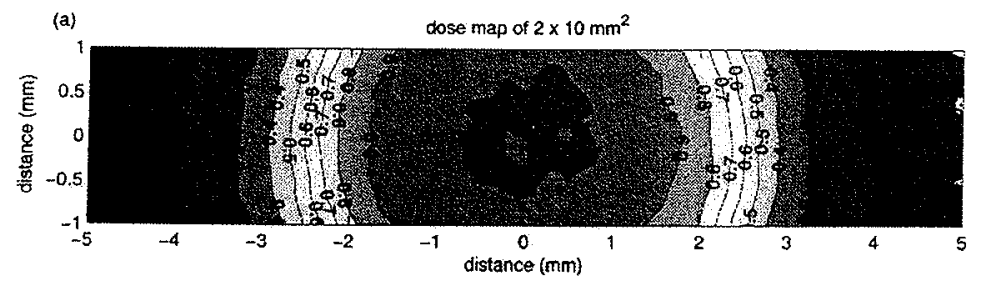

Fig. 14. Dose map of (a) $2 \times 10 \mathrm{~mm}^{2}$ and (b) 1

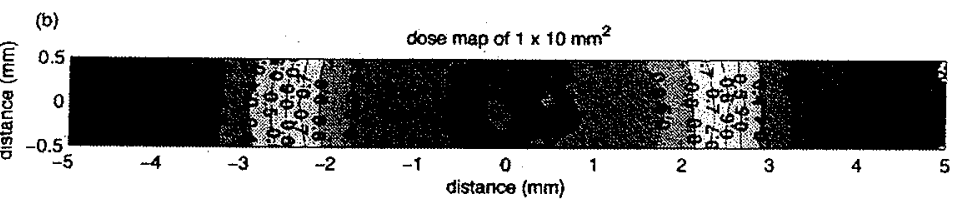

profile, the effect of volume averaging for the $1 \times 1 \mathrm{~mm}^{2}$ detector relative to the $0.2 \times 2 \mathrm{~mm}^{2}$ detector is insignificant in comparison to the volume averaging effects within the penumbra region.

To summarize, the size of the detector is important in small field dosimetry. A detector with larger dimensions will tend to cause volume averaging of the dose. However, when considering detectors with respective sizes of $1 \times 1$ and 0.2 $\times 2 \mathrm{~mm}^{2}$, the effect of detector volume averaging should be considered for specific beam geometry (beam diameter, detector diameter, and region of measurement) and the purpose of the measurement. In the case of a $5 \mathrm{~mm}$ diameter circular beam with radial symmetry, measurement of the center of the beam such as the total scatter factor, $S_{\mathrm{cp}}$, a difference in the longitudinal dimension of $1 \mathrm{~mm}$ versus $2 \mathrm{~mm}$ would produce only a small discrepancy since over most of the region the beam profile does not have a high dose gradient. However, if the same detectors were to be used for measurement within the penumbra region where there is a steep dose gradient, a detector with $0.2 \times 2 \mathrm{~mm}^{2}$ would be more advantageous than a detector with $1 \times 1 \mathrm{~mm}^{2}$ width because the dose gradient in the transverse direction is significantly steeper than that in the longitudinal direction.

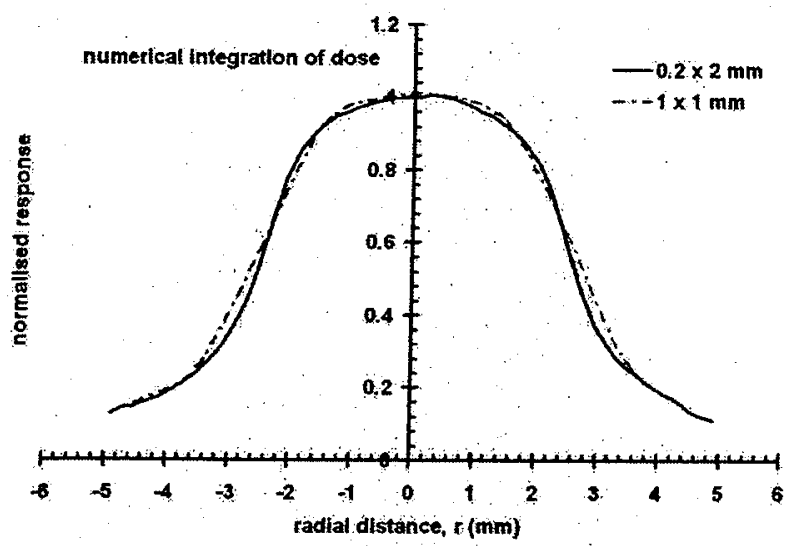

FIG. 15. Numerical integration of dose for detector with areas of $0.2 \times 2$ and $1 \times 1 \mathrm{~mm}^{2}$.

\section{CONCLUSION}

A Si strip detector DMG was successfully evaluated for its dose per pulse and angular dependence. It has been tested for typical SRS dose deliveries. A custom-made SRS phantom was made that allows accurate and reproducible setups using a commercially available SRS couch mount. The change in the dose per pulse response of the DMG is less than $5 \%$ and $11 \%$ for dose per pulse factor changes of 7.5 and 22 , respectively. The maximum polar angular response was $13.8 \% \pm 0.1 \%$ at a gantry angle of $320^{\circ}$ when the couch position was at $270^{\circ}$. This may be due mainly to geometrical artifacts rather than the detector's response. The maximum azimuth angular response was $15.3 \% \pm 0.1 \%$ at gantry angles of $90^{\circ}$ and $270^{\circ}$. The improved angular response is partly due to better detector packaging, which was mounted on a $0.12 \mathrm{~mm}$ Kapton substrate. The DMG was used to measure the isocenter displacement due to gantry, collimator, and couch rotations of the linear accelerator. The overall positioning uncertainty in the DMG due to couch, gantry, collimator rotation, and the positioning of the SRS cones was found to be $\pm 0.8 \mathrm{~mm}$. This translates to an uncertainty in the measurement of $\pm 2 \%$ for the smallest cone diameter. The

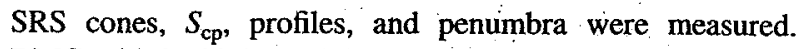
DMG with its high spatial resolution of $0.2 \mathrm{~mm}$ was able to capture the dose profile of the smallest SRS cone of $5 \mathrm{~mm}$ in diameter. The maximum difference between DMG and EBT2 film measurements for the cone $S_{\mathrm{cp}}$ values was $3.8 \%$ for cone diameter of $5 \mathrm{~mm}$. We also tested the DMG in SRS arc delivery where four noncoplanar SRS arcs of $0^{\circ}-180^{\circ}$ were delivered with different couch angles. The maximum difference in profiles between the DMG and the EBT2 film was less than $2.5 \%$. The novelty of the DMG combines the advantage of multiple single element detectors in measuring a small radiation field. In addition, the real time acquisition of the DMG allows quick evaluation of SRS dose profiles.

\section{ACKNOWLEDGMENTS}

One of the authors (J. H. D. Wong) was supported by the Public Institution of Higher Learning Academic Training. Scheme (SLAI), Ministry of Higher Education, Malaysia. The authors would like to thank Professor Roberto Cirio and 
Dr. Marco Donetti, Turin INFN for long term collaboration and advice on TERA chip DAQ system. One of the authors (P.M.) acknowledges financial support from the Cancer Institute of NSW clinical leaders program. The author would like to acknowledge Sam Khanna for his contribution in the development of the data acquisition software, Karl Chan for the Monte Carlo calculation of the $S_{\mathrm{cp}}$ values, and Dr. Phil Simmonds for proofreading the manuscript. The authors would like to thank Peter Ihnat, Ron Marshall, and Martin Morillas from UoW for the technical support rendered in the project. The authors would like to thank the Australian Institute for Nuclear Science and Engineering (AINSE) for their support in the research and development of the DMG detector under Award No. AINGRA08039. The authors would like to thank the reviewer for the suggested formalism for angular response correction [Eq. (8)], which was a refinement of the original formalism.

\footnotetext{
a) Author to whom correspondences should be addressed. Electronic mail: anatoly@uow.edu.au

${ }^{1}$ A.-L. Grosu, P. Kneschaurek, and W. Schlegel, in New Technologies in Radiation Oncology, edited by W. Schlegel, T. Bortfeld, and A.-L. Grosu (Springer-Verlag, Berlin, 2006), pp. 267-276.

${ }^{2} \mathrm{P}$. Metcalfe, T. Kion, and P. Hoban, The Physics of Radiotherapy X-Rays and Electrons (Medical Physics, Madison, WI, 2007)

${ }^{3}$ M. Heydarian, P. W. Hoban, and A. H. Beddoe, "A comparison of dosimetry techniques in stereotactic radiosurgery," Phys. Med. Biol. 41(1), 93110 (1996).

${ }^{4}$ I. J. Das, G. X. Ding, and A. Ahnesjo, "Small fields: Nonequilibrium radiation dosimetry," Med. Phys. 35(1), 206-215 (2008):

${ }^{5}$ A. S. Beddar, D. J. Mason, and P. F. O'Brien, "Absorbed dose perturbation caused by diodes for small field photon dosimetry," Med. Phys. 21(7), 1075-1079 (1994).

${ }^{6}$ A. J. D. Scott, A. E. Nahum, and J. D. Fenwick, "Using a Monte Carlo model to predict dosimetric properties of small radiotherapy photon fields," Med. Phys. 35(10), 4671-4684 (2008).

${ }^{7}$ A. J. D. Scott, A. E. Nahum, and J. D. Fenwick; "Monte Carlo modeling of small photon fields: Quantifying the impact of focal spot size on source occlusion and output factors, and exploring miniphantom design for small-field measurements," Med. Phys. 36(7), 3132-3144 (2009).

${ }^{8}$ E. Pappas, T. G. Maris, F. Zacharopoulou, A. Papadakis, S. Manolopoulos, S. Green, and C. Wojnecki, "Small SRS photon field profile dosimetry performed using a PinPoint air ion chamber, a diamond detector, a novel silicon-diode array (DOSI), and polymer gel dosimetry. Analysis and intercomparison," Med. Phys. 35(10), 4640-4648 (2008).

${ }^{9} \mathrm{~W}$. U. Laub and T. Wong, "The volume effect of detectors in the dosimetry of small fields used in IMRT," Med. Phys. 30(3), 341-347 (2003).

${ }^{10} \mathrm{~F}$. García-Vicente, M. J. Béjar, L. Péreż, and J. J. Torres, "Clinical impact of the detector size effect in 3D-CRT," Radiother. Oncol. 74(3), 315-322
}

(2005)

"L. Paelinck, W. D. Neve, and C. D. Wagter. "Precautions and strategies in using a commercial flatbed scanner for radiochromic film dosimetry," Fhys. Med. Biol. 52(1), 231-242 (2007)

${ }^{12} \mathrm{M}$. J. Butson, T. Cheung, and P. K. Yu, "Scanning orientation effects on Gafchromic EBT film dosimetry," Australas. Phys. Eng. Sci. Med. 29(3), 281-284 (2006)

${ }^{13}$ M. J. Butson, P. K. N. Yu, T. Cheung, and P. Metcalfe, "Radiochromic film for medical radiation dosimetry," Mater. Sci. Eng. R. 41(3-5), 61120 (2003).

${ }^{14}$ R. K. Rice, J. J. Hansen, G. K. Svensson, and R. L. Siddon, "Measurements of dose distributions in small beams of $6 \mathrm{MV}$ x-rays," Phys. Med. Biol. 32(9), 1087-1099 (1987).

${ }^{15}$ K. Vergote, Y. D. Deene, W. Duthoy, W. D. Gersem, W. D. Neve, E. Achten, and C. D. Wagter, "Validation and application of polymer gel dosimetry for the dose verification of an intensity-modulated are therapy (IMAT) treatment," Phys. Med. Biol. 49(2), 287-305 (2004).

${ }^{16}$ J. H. D. Wong, M. Carolan, M. L. F. Lerch, M. Petasecca, S. Khanna, V. L. Perevertaylo, P. Metcalfe, and A. B. Rosenfeld, "A silicon strip detector dose magnifying glass for IMRT dosimetry," Med. Phys. 37(2), 427$439(2010)$.

${ }^{17}$ S. M. Sze, Semiconductor Devices: Physics and Technology, 2nd ed. (Wiley, New York, 2001).

${ }^{18}$ P. D. Higgins, P. Alaei, B. J. Gerbi, and K. E. Dusenbery, "In vivo diode dosimetry for routine quality assurance in IMRT," Med. Phys. 30(12), 3118-3123 (2003).

${ }^{19} \mathrm{D}$. Marre and G. Marinello, "Comparison of p-type commercial electron diodes for in vivo dosimetry," Med. Phys. 31(1), 50-56 (2004).

${ }^{20} \mathrm{M}$. Westermark, J. Andt, B. Nilsson, and A. Brahme, "Comparative dosimetry in narrow high-energy photon beams," Phys. Med. Biol. 45(3), 685-702 (2000).

${ }^{21}$ P. A. Jursinic, "Angular dependence of dose sensitivity of surface diodes," Med. Phys. 36(6), 2165-2171 (2009).

${ }^{22}$ M. J. Berger, J. S. Coursey, M. A. Zucker, and J. Chang, Stopping-Power and Range Tables: Electrons, Protons, Helium Ions [webpage] (accessed September 1, 2009), available at http://physics.nist.gov/PhysRefData/ Star/Text/contents.html.

${ }^{23} \mathrm{~S}$. Wellhöfer, User's Guide to the SP34 QA Phantom (2003).

${ }^{24} \mathrm{I}$ ' $m$ RT phantom accessed available at http://iba-dosimetry.com/I-m-i-RTi-Phantom.451.0.hml.

${ }^{25}$ Varian Oncology Systems, Clinac 2100 CD, 2300 CDD, 21EX, 23EX System Manual (Varian Associates, Inc., Oncology Systems, United States, 1998).

${ }^{26}$ M. Fuss, E. Sturtewagen, C. D. Wagter, and D. Georg, "Dosimetric characterization of GafChromic EBT film and its implication on film dosimetry quality assurance," Phys. Med. Biol. 52(14), 4211-4225 (2007).

${ }^{27}$ J. E. Matney, B. C. Parker, D. W. Neck, G. Henkelmann, and I. I. Rosen, "Evaluation of a commercial flatbed document scanner and radiographic film scanner for radiochromic EBT film dosimetry," J: Appl. Clin. Med. Phys. 11(2), 198-208 (2010).

${ }^{28}$ W. Lutz, K. R. Winston, and N. Maleki, "A system for stereotactic radiosurgery with a linear accelerator," Int. J. Radiat. Oncol., Biol., Phys. 14(2), 373-381 (1988). 
